\title{
Optimization of the drayage problem using exact methods
}

Reinhardt, Line Blander; Pisinger, David; Spoorendonk, Simon; Sigurd, Mikkel M.

Published in:

I N F O R Journal

Link to article, DOI:

10.1080/03155986.2016.1149919

Publication date:

2016

Document Version

Peer reviewed version

Link back to DTU Orbit

Citation (APA):

Reinhardt, L. B., Pisinger, D., Spoorendonk, S., \& Sigurd, M. M. (2016). Optimization of the drayage problem using exact methods. I N F O R Journal, 54(1), 33-51. https://doi.org/10.1080/03155986.2016.1149919

\section{General rights}

Copyright and moral rights for the publications made accessible in the public portal are retained by the authors and/or other copyright owners and it is a condition of accessing publications that users recognise and abide by the legal requirements associated with these rights.

- Users may download and print one copy of any publication from the public portal for the purpose of private study or research.

- You may not further distribute the material or use it for any profit-making activity or commercial gain

- You may freely distribute the URL identifying the publication in the public portal

If you believe that this document breaches copyright please contact us providing details, and we will remove access to the work immediately and investigate your claim 


\title{
Optimization of the drayage problem using exact methods
}

\author{
Line Blander Reinhardt*a , David Pisinger ${ }^{\mathrm{a}}$, Simon Spoorendonk ${ }^{\mathrm{a}}$ and Mikkel M. Sigurd ${ }^{\mathrm{b}}$ \\ a Department of Management Engineering, Technical University of Denmark, \\ Produktionstorvet, Building 426, DK-2800 Kgs. Lyngby, Denmark \\ ${ }^{\mathrm{b}}$ Maersk line, Esplanaden 50,DK-1098 Copenhagen K, Denmark
}

\begin{abstract}
Major liner shipping companies offer pre- and end-haulage as part of a door-to-door service. However, pre- and end-haulage is one of the major bottlenecks in liner shipping due to the lack of coordination between the customers often leading to inefficiency. In this paper we apply techniques from vehicle routing problems to the problem of pre- and end-haulage of containers, and test it on data from a major liner shipping company. The paper contains several versions of the problem such as multiple empty container depots, and balancing the empty container depot levels and their influence on the cost is shown. Finding a set of optimal vehicle routes allowing these combinations is NP-hard. However, by exploring the fact that the number of possible routes in the considered case is quite limited, we show that the model can be solved within a minute by using column enumeration. Alternative constraints and problem formulations are considered. Computational results are reported on real-life data from a major liner shipping company.
\end{abstract}

Keywords: Triangulation, Drayage, Liner shipping, Vehicle routing, Column enumeration, Open requests

\section{Introduction}

The pre- and end-haulage of containers is an important part of the service provided by a liner shipping company. Whereas the liner ships can carry several thousand containers, the pre- and end- haulage is the transport of the individual container from their arrival terminal to the customer. In this paper we consider pre- and end-haulage for a European country and both problem and data are real-life situations of one of the leading liner shipping companies in the world. The results presented here are implementation and test results of the modeling ideas discussed in the conference paper [9].

Reducing the number of kilometers traveled will not only save the shipping or trucking companies money but also reduce the $\mathrm{CO}_{2}$ emission.

In the literature pre- and end-haulage is also referred to as inland container transport, hinterland service or drayage. Pre- and end-haulage start at the sea ports, the river beds or dry ports at

*email: lbre@dtu.dk,tel: ++45 45253389 
the start of the day. The containers are transported to the river bed and dry ports by barge, truck or by train. All of these locations are in this paper referred to as terminals. Here we only look at the transport by truck to and from terminals. In the considered problem there are several terminals and all terminals have container yards for both full and empty containers.

The orders are denoted as import and export orders. Even though the transport by truck often is served by subcontractors the transported containers are usually owned by the liner shipping companies. Therefore the transport of empty containers is considered part of fulfilling the import and export orders. At the terminals there are cranes which can move a container on to the truck, such cranes are not available at the costumers and the truck must wait with the container at the costumer until the container is emptied or filled. For an export order an empty container is transported to the customer where it is filled and the full container is then delivered at the departure terminal. For an import order a full container must be delivered from the arrival terminal to the customer. At the customer location the import container is emptied and the empty container is either taken to a terminal for stowage, or to an export customer. The task of filling or emptying a container at the customer location is in this paper referred to as a service.

The use of an import container for an export order is defined as triangulation of two orders.

An example of single and multi depot triangulations can be seen in Figure 1. Triangulating the orders can reduce the cost by reducing the total distance traveled by the trucks and the total gate entry cost to be paid. However, a possible wait time before the export customer can be serviced will introduce a waiting cost which will increase the overall cost. Another cost is the gate entry fee at the terminals which is paid every time a truck enter a terminal. Thus there is a possible saving in following an import order by an export order so that empty containers are put to use immediately.

Terminal

$\longleftarrow$ Full container

- Export customer $\leftarrow--$ Empty container

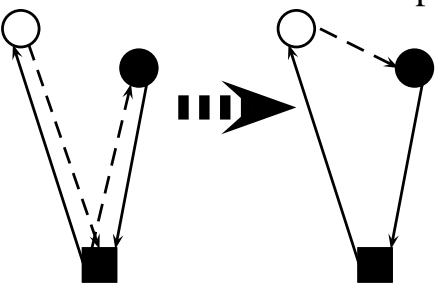

(a) Standard triangulation.

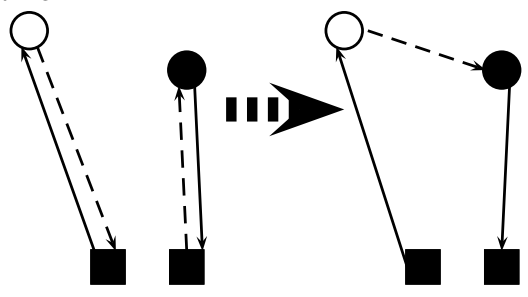

(b) Multi depot triangulation.

Figure 1: Figures (a) and (b) show triangulations of import and export orders.(a) shows the triangulation of an import trip and an export trip both connected to the same terminal. (b) shows the triangulation of an import trip and an export trip belonging to two different terminals.

The most common scenario is that a single customer is serviced per container. However, it may happen that a container is filled at multiple customer locations in case of an export order or emptied at multiple customer locations in case of an import order. When multiple customers share a container, then the goods are ordered in vertical slices corresponding to each customer. 
This arrangement of goods within the container also determines the order in which the customers should be visited. Containers serviced at multiple locations are called multi stop orders. Since the order of the customer visits is predetermined for a multi stop order the journey between the first and the last customer is fixed and the triangulation can only occur after the visit to the last customer is finished for an import order or before the visit to the first customer is started for an export order.

Another type of triangulation is the chassi-triangulation where the truck can leave the trailer, that carries the container, at the customer for service (filling or emptying). A (possibly different) truck will then be assigned to pick up the chassi with the container after the service has been completed. This may give the truck time for other deliveries while the container is being serviced at a customer location. Chassi-triangulations are to some extent practiced in North America. However, in the rest of the world the trucks are usually not able to leave the trailer behind. This can be due to physical limitation of the truck or due to the fact that interchanging a trailer between two trucks requires the trucks to be of the same type. Moreover two different trucking companies will usually not be willing to exchange trailers. The paper by Imai et al. [4] confirms this limitation for pre- and end-haulage in Japan.

In a real-life subcontracted pre- and end-haulage problem, a route starts the moment a container is placed on the truck at a terminal and ends the moment the container is removed from the truck (at a terminal). The liner shipping company then only buys a transport of a container and as soon as the container is removed from the vehicle the subcontracted vehicle is free to do other jobs which may be from other companies. Therefore from the liner company perspective the number of routes is not desired to be minimized in the real-life problem. However the problems presented in the literature often represent the viewpoint of the trucking companies and therefore the available vehicles are often limited or the number of vehicles used is minimized due to operational cost for each additional vehicle. To include this aspect we consider a version of the problem where the number of vehicles is minimized.

In this paper the transport of empty containers (also called repositioning) from one terminal to another is not included. Clearly, if the vehicles always transport the empty containers to and from the closest container yards then the distance the empty containers are transported is minimized. This, however, may result in some container yards overflowing with empty containers while others do not have enough empty containers to satisfy the demand. This may result in the necessity of repositioning empty containers which leads to additional travel distances. Therefore, to satisfy the demand for empty containers the liner shipping companies may have requirements for where to store the empty containers. Such balance requirements placed on the terminals are considered in our model.

The liner shipping company studied has predefined the pickup and drop off locations for empty containers to ensure that there is no shortage of empty containers or storage space. This, however, may result in longer driving distances than needed as empty containers may be stored at locations further away. Moreover, when triangulating orders, the container is not stored at or removed from the predefined locations so imbalances may still occur.

A way to solve this could be to put restrictions on the imbalance of empty containers at the individual container yards and possibly punish any imbalances. In this paper we formulate and test constraints which restrict the difference in the inventory levels of empty containers at the terminals. The difference in the inventory level at a terminal is calculated as the difference between 
the inventory level before the orders are initiated and after all orders are finished.

The remainder of the paper is organized as follows: Section 2 contains a brief introduction to the existing literature on the topic. In Section 3 the mathematical model and its extensions are presented with the solution method. In Section 4.1 the test instances are presented and in Section 4.2 the test results are presented. The findings of the paper are discussed and conclusions are made in Section 5.

\section{Literature review}

In recent years the literature on pre- and end-haulage of container transport has increased significantly. However, there are many different versions of the problem. To provide an overview Table 1 lists the problem properties presented in some of the most recent papers on pre- and end-haulage. The table is based on the overview presented by Schönberger et al. in [10], extended with additional algorithm properties. In the table there is a column which shows the solution method used. To clarify the term "depot" in the table it should be noted that in the routing literature the term depot usually refers to the location where the journey of the vehicles starts and ends. In container transport the depot often refers to the location where empty containers are stored. In Table 1, depot refers to the storage location of empty containers. In some problems the terminal has a yard for storing empty containers and therefore is considered both a terminal and a depot.

Many papers assume that the container can be removed from the truck at the customer location, (Braekers et al. [1], [2], Jula et al. [5], Nossack and Pesch [7], Schönberger et al. [10], Vidovic et al. [12], Wang and Yun [13], Wang and Regan [14], Zhang et al. [15] and [16]). However, it is noted by Imai et al. [4] that this is only the case when the chassi can be removed from the truck. It is very rare that the individual customer has a crane for lifting the container off the truck, and such customers will often have their own train terminal or barge port, and therefore will not be part of the considered problem. Chassi triangulation is rarely practiced outside North America. In Imai et al. [4] and Caris and Janssens [3] the container follows the truck. This property is presented in the $6^{\text {th }}$ column in Table 1 entitled "drop off".

Imai et al. [4] formulated the problem of routing vehicles for container orders with linkage between pickup and delivery, as a full truck load version of the vehicle routing problem with backhauls (empty containers). Imai et al. [4] solved the problem using lagrangian relaxation based heuristic. For details on the vehicle routing problem with backhauls see e.g., [6],[8] and [11].

The solution method used by Braekers et al. [1] is the deterministic annealing heuristic. Zhang et al. in [16] use a heuristic to solve instances with up to 895 loads and in [15] a two stage approximation approach to solve a stochastic version of their version of the drayage problem with up to 75 tasks.

Clearly, since the delivery location must immediately follow the pickup location for full truck loads this problem can also be considered as the asymmetric multiple traveling salesman problem by merging the pickup and delivery of a container order into one node (see Jula et al. [5], Wang and Regan [14] and Zhang et al. [16]). As noted by Zhang et al. [16] the pre- and end-haulage problem differs from the problems in [5], [14] and [16] by the fact that it has the empty container requests for which either the pickup or delivery location is not fixed but may be selected among 


\begin{tabular}{|c|c|c|c|c|c|c|c|c|c|c|}
\hline Literature & load & depots & terminals & TW & $\begin{array}{c}\text { drop } \\
\text { off }\end{array}$ & $\begin{array}{c}\text { open } \\
\text { requests }\end{array}$ & $\begin{array}{l}\text { vehicles } \\
\text { limited }\end{array}$ & objective & $\begin{array}{c}\text { solution } \\
\text { method }\end{array}$ & $\begin{array}{c}\text { number of } \\
\text { orders }\end{array}$ \\
\hline Wang and Regan 2002 [14] & FT & $\mathrm{m}$ & $\mathrm{m}$ & yes & yes & no & yes & min cost $/$ max orders & heuristic & up to 75 \\
\hline Jula et al. 2005 [5] & FT & $\mathrm{m}$ & $\mathrm{m}$ & yes & yes & no & yes & $\min \operatorname{cost}$ & $\begin{array}{c}\text { exact } \\
\text { heuristic }\end{array}$ & $\begin{array}{l}\text { up to } 20 \\
\text { up to } 100\end{array}$ \\
\hline Imai et al. 2007 [4] & FT & 1 & 1 & no & no & yes & yes & $\min$ cost & heuristic & up to 200 \\
\hline Caris and Janssens 2009 [3] & FT & 1 & 1 & yes & no & yes & yes & $\min \operatorname{cost}$ & heuristic & up to 200 \\
\hline Zhang et al. 2010 [16] & FT & $\bar{m}$ & $\bar{m}$ & yes & yes & yes & yes & min time & heuristic & up to 75 \\
\hline Braekers et al. 2011 [1] & FT & $\mathrm{m}$ & $\mathrm{m}$ & yes & yes & yes & no & $\begin{array}{l}\text { bi-objective: } \\
\text { min vehicles } \\
\text { min distance }\end{array}$ & heuristic & up to 200 \\
\hline Vidovic et al. 2011 [12] & LTL & 1 & 1 & no & yes & no & no & $\min \cos t$ & $\begin{array}{c}\text { exact } \\
\text { heuristic }\end{array}$ & $\begin{array}{l}\text { up to } 36 \\
\text { up to } 36\end{array}$ \\
\hline Zhang et al. 2011 [15] & FT & $\mathrm{m}$ & $\mathrm{m}$ & yes & yes & yes & yes & $\min \operatorname{cost} / \mathrm{max}$ orders & heuristic & up to 75 \\
\hline Reinhardt et al. 2012 [9] & FT & $\mathrm{m}$ & $\mathrm{m}$ & yes & no & yes & no & min cost & exact & up to 305 \\
\hline Braekers et al. 2013 [2] & FT & $\mathrm{m}$ & $\mathrm{m}$ & yes & yes & yes & yes & $\begin{array}{l}\text { bi-objective: } \\
\text { min vehicles } \\
\text { min distance }\end{array}$ & heuristic & up to 200 \\
\hline Wang and Yun 2013 [13] & FT & 1 & $\mathrm{~m}$ & yes & yes & yes & yes & $\min$ cost & heuristic & up to 40 \\
\hline Nossack and Pesch 2013 [7] & FT & $\mathrm{m}$ & $\mathrm{m}$ & yes & yes & yes & yes & min total time & heuristic & up to 75 \\
\hline Schönberger et al. 2013 [10] & LTL & 1 & 1 & no & yes & yes & yes & min distance & exact & up to 6 \\
\hline This paper & FT & $\mathrm{m}$ & $\mathrm{m}$ & yes & no & yes & no & $\begin{array}{c}\text { min cost } \\
\text { min vehicles }\end{array}$ & exact & up to 308 \\
\hline
\end{tabular}

Table 1: Properties of recent models for pre- and end-haulage. FT and LTL stand for Full Truck load and Less Than full truck Load (up to 2 twenty foot containers per truck load) respectively in the column load. The depot is the location where empty containers are located. The number of terminals or depots in the problem is 1 for single and $\mathrm{m}$ for multiple. The terminal is an intermodal connection point where the containers are shipped to or from with mass transportation such as liner vessels, barges or trains. Time windows are denoted by TW. Open requests indicates if a the origin of the empty container is open or fixed. When No the origin is fixed. vehicles limited is yes if there is a limited number of vehicles available. In the column objective the objective function is described. In the column solution method it is indicated if an exact or a heuristic method has been applied. The column number of orders indicates the size of the instances solved. 
several terminals or customers in need of empty containers. Empty container orders with open destination or origin are also called open requests [10]. An open request can be considered an independent request if it is assumed that the container is removed from the truck at the customer location. When containers cannot be removed from the truck at the customer location the empty container request is related to a full container request. In both cases the empty container requests are considered open requests. In this paper we model open empty container request and fixed empty depot request and compare the solutions.

The problem of full truckload pickup and delivery with hard time windows was described and solved by Wang and Regan [14] in 2002 by an exact method. Later in [5] Jula et al. solved the problem describe by Wang and Regan [14] by an exact method for smaller instances with up to 15 locations and a genetic algorithm for large instances with up to 895 locations. Note that in both [5] and [14] the open requests are not considered.

Imai et al. [4] note that in the case where each route handles exactly one order the problem becomes an assignment problem and can be solved in polynomial time using the Hungarian method. However, when the routes may contain more than one order, the problem becomes a generalized assignment problem which is known to be NP-hard. This is described in more detail in [4] where a proof of NP-hardness for the vehicle routing problem with full container loads (VRPFC) is presented.

Since the VRPFC problem is NP-hard many different heuristic methods have been presented to solve the problem. Imai et al. [4] solve the VRPFC without time windows using a heuristic method based on Lagrangian relaxation. The problem solved by Imai et al. in [4] only has one depot and has open requests. Clearly a problem with open requests is more complex than a problem where the requests are fixed in both ends. However when the problem only has one depot the number of possible locations is reduced resulting in a less complicated problem.

Caris and Janssens [3] extended the problem described by Imai et al. [4] by adding time windows, and they solved the problem using a two-phase insertion heuristic. Reinhardt et al. [9] used an exact method to solve the problem with time windows and pickup and storage of the empty containers at the closest location.

The remaining papers consider the variant of VRPFC where containers are removed from the truck at the customer location. As mentioned earlier this assumption very rarely represents the real life situation.

Wang and Yun [13] include the train arrival schedule in the problem and solve instances with up to 40 orders using a hybrid tabu search. Vidovic et al. [12] allow two 20ft containers to be transported on one truck and solve the problem by exact and heuristic methods for instances with up to 36 orders. For some of these the exact method used hours to find a solution. Schönberger et al. [10] as well as Vidovic et al. [12] consider the problem where two 20ft containers may be carried on the same truck (LTL) and solve the problem for instances with up to 6 orders by an exact method. In this paper the possibility of two $20 \mathrm{ft}$ containers on a truck is not considered. This is due to the fact that containers can not be removed from the truck at customer locations and therefore as noted by the liner shipping company one container will block for the access to the goods in the other container.

The formulation presented in this paper is mostly related to the formulation of Imai et al. [4] and Caris and Janssens [3]. However, contrary to the limited number of vehicles in [3] and [4], there is no restriction on the number of vehicles used in the problem presented here. Braekers et 
al. [1] and Vidovic et al. [12] also consider problems without limits on the number of vehicles.

In this paper we solve the pre- and end-haulage of a liner shipping company using column enumeration. In the column enumeration each column is a route represented by a binary column variable. An optimal covering of all orders is found by using integer programming. The problem formulation is similar to Braekers et al. [1], Caris and Janssens [3] and Imai et al. [4]. The model is very similar to the preliminary studies presented in Reinhardt et al. [9] with extension on the minimization of the number of vehicles and testing of suggested models.

The considered test instances are based on real-life operations and the assumptions presented here reflect the criteria of the shipping company. We show that even though many orders must be satisfied every day it is not difficult to enumerate all possible routes as the real-life distances, handling time, container match and time windows restrict the number of feasible routes significantly.

\section{Mathematical Model}

In this section we present a model for the pre- and end-haulage problem of a liner shipping company. In the considered problem multiple terminals and depots are allowed. All customers have an associated time window. We model and solve several different versions where the depot for picking up or storing the empty container can be selected freely or is fixed by the company. Only one container is allowed to be on a truck at a time and the container follows the truck until a depot or terminal is visited. The model presented in this paper can handle both the cases where the chassi can be removed and where the container stays with the truck at customer locations. However, since the test data is from a country where the removal of chassis is not practiced the tests only represent the case where a container can solely can be removed from a truck at a terminal.

We here present a path formulation of the problem, hence the problem is decomposed into two parts: 1) finding all feasible routes, 2) selecting a set of routes so that all the orders are covered at a minimal cost, which is also called the set covering problem.

\subsection{The order covering model}

The problem is formulated as a set covering problem which combines the routes so that the orders are completed at a minimal cost. Let the sets:

- $P$ be the set of all possible routes (trip patterns),

- $K$ be the set of all orders

and let the parameters:

- $c_{p}$ be the cost of a path $p \in P$,

- $\alpha_{k p}$ be 1 if path $p \in P$ covers order $k \in K$ (move of full container) and zero otherwise,

- $\beta_{k p}$ be 1 if path $p \in P$ covers the move of the empty container related to order $k \in K$ and zero otherwise,

- $O_{k}$ be the demand for order $k \in K$ (there can only be one container per order as a truck can at most carry one container), 
- $a_{k p}$ be the arrival time of the vehicle on the path $p \in P$ at the customer related to order $k \in K$,

- $d_{k p}$ be the departure time of vehicle on the path $p \in P$ at the customer related to order $k \in K$,

- $s$ be the time it takes to fill or empty a container.

Moreover, let the variables:

- $y_{p}$ be a binary variable which is 1 if path $p \in P$ is used and zero otherwise.

The parameters $a_{k p}$ and $d_{k p}$ are only needed in the case where chassi-triangulations are possible so that the empty and full container part of an order can belong to two different routes.

The problem can be formulated as the following binary program:

$$
\begin{aligned}
\text { IP1: } \min & \sum_{p \in P} c_{p} y_{p} \\
\text { s.t. } & \sum_{p \in P} \alpha_{k p} y_{p}=O_{k} \\
& \sum_{p \in P} \beta_{k p} y_{p}=O_{k} \\
& \sum_{p \in P} a_{k p} y_{p}+s \leq \sum_{p \in P} d_{k p} y_{p} \\
& y_{p} \in\{0,1\}
\end{aligned}
$$

The objective (1) is to minimize overall cost of the routes. Constraints (2) ensure that all orders are completed, constraints (3) ensure that all empty container pickup and deliveries related to the orders are completed, and constraints (4) ensure that for export orders an empty container has arrived so that the container can be filled before being picked up, and for imports that the full container has arrived before the empty is picked up. Constraints (5) define the variable domain. If chassi-triangulation patterns are not considered and the container must stay with the vehicle at the customers, then constraints (4) are redundant. Moreover, when not allowing chassitriangulations then the constraints (3) are not needed as delivery and return of the empty container would be completed in the same trip as the order (full container transport). Note that in the case of chassi-triangulations the container maybe delivered and picked up on two different paths and the constraints (4) and (3) are therefore needed. The set $P$ contains all feasible paths. The generation of these paths is described in the following subsection.

\subsection{Enumeration of all feasible paths}

Path generation is done by full enumeration of all feasible paths. In the cases where the number of vehicles is not minimized the enumerated paths contains all feasible combinations of an import and an export order. Note that the number of paths in this case is polynomial when the depot for the empty container of the order is predetermined. In the case where the empty container depot is 
open the optimal solution selects the closest depot. When a balance restriction is put on the depot a path using each empty container depot must be generated for each order.

In the case described in IP1 where the number of vehicles is not minimized, the paths can be found by finding all single-location paths and all possible triangulations. A single-location path for an export order is to bring an empty container from a terminal to the customer, fill it, and then bring the full container to the departure terminal. A single-location path for an import order is to bring the full container to a customer, empty it and then bring the empty container to a (predefined or open) storage location. Note that a path represents the journey of a vehicle carrying a container. This journey ends when the vehicle returns to a depot or terminal. In order to find all (non-chassi) triangulation paths we combine all import orders with all export orders and verify the feasibility of the path, i.e, bringing a full container to an import customer, empty the container, then bring the empty container to an export customer, fill the container, and then bring the full container to the destination terminal. The feasibility is verified by checking whether the container types can be matched and the time window is satisfied.

When the number of vehicles is minimized the paths can be a combination of more than two orders. We have included the case where the depot for the empty container is predetermined (but can be violated by triangulating an import and export order) and the case where the empty container can be dropped off at a depot where a full container is picked up or an empty container can be picked up for the depot where a full container was dropped off by the same vehicle.

Chassi-triangulation is not considered in the computational experiments since this type of triangulation is not possible in the real-life data instances used for the tests.

\subsection{Minimizing the number of vehicles}

Let $Q$ be the set of vehicle routes. This set of routes consists of all routes in the set $P$ (which is the container routes) and all feasible combinations of the routes in $P$ with respect to time windows and duration. The routes in $P$ can be combined by creating a connecting trip from the end location of one route to the start location of the next route. Since the distances in the data provided are quite large and the time windows tight, this does not result in an unreasonable amount of paths. However, if the time windows were loose and the distances were small this could result in a huge amount of paths. When reducing the number of vehicles the objective (1) of IP1 must be changed to the following:

$$
\text { IP2: } \quad \min \quad \sum_{q \in Q}\left(c_{q}+C\right) y_{q}
$$

where $C$ is the penalty for using a vehicle. It could also be a known cost of using a vehicle. Note that the constraints will be the same as constraints (2) to (5) where $p$ and $P$ are replaced by $q$ and $Q$, respectively. We include the cost of the paths $c_{q}$ in the objective function since we wish to minimize the total path cost given a minimal number of vehicles. The constant $C$ should be chosen larger than any $c_{q}$. The number of vehicles then is sure to be minimal. However it is a weighted function of multiple criteria. Since it in this case is important to always have the minimal number of vehicles the parameter $C$ can be selected using the input (as this may vary). 


\subsection{Container yard balancing}

As mentioned earlier it may not be possible to pickup or deposit empty containers at the nearest terminal. Requirements on the balance of empty containers may apply to the terminals. These requirements can be specific for a given day due to resource limitations. This can be relevant as empty container repositioning (between terminals) is not included in the problem. By introducing container yard balance requirements it is possible to incorporate information which will ensure that empty containers are transported to container yards lacking containers and empty containers are retrieved from container yards overflowing with containers. To introduce the container yard balance constraints we let:

- $T$ be the set of all terminals,

- $B_{t a} \geq 0$ be the allowed increase in empty container level at terminal $t \in T$,

- $B_{t b} \leq 0$ be the allowed decrease in empty container level at terminal $t \in T$,

- $\psi_{t p_{+}}$be the number of empty containers returned to terminal $t \in T$ on a path $p \in P$,

- $\psi_{t p_{-}}$be the number of empty containers taken from terminal $t \in T$ on a path $p \in P$.

Note that the parameters $\psi_{t p_{+}}$and $\psi_{t p_{-}}$are determined when generating the route $p$ just as the parameters $\alpha_{k p}$ and $\beta_{k p}$. The depot balance constraints can be added to the problem by introducing the following constraints:

$$
\begin{array}{rlr}
\text { IP3: } \min & \sum_{p \in P} c_{p} y_{p} & \\
\text { s.t. } & \sum_{p \in P} \alpha_{k p} y_{p}=O_{k} & k \in K \\
& \sum_{p \in P} \beta_{k p} y_{p}=O_{k} & k \in K \\
& \sum_{p \in P} a_{k p} y_{p}+s \leq \sum_{p \in P} d_{k p} y_{p} & k \in K \\
& \sum_{p \in P}\left(\psi_{t p_{+}} y_{p}-\psi_{t p_{-}} y_{p}\right) \leq B_{t a} & t \in T \\
& \sum_{p \in P}\left(\psi_{t p_{+}} y_{p}-\psi_{t p_{-}} y_{p}\right) \geq B_{t d} & t \in T \\
& y_{p} \in\{0,1\} &
\end{array}
$$

This gives a lower and upper bound on how much the balance may differ at terminal $t$ from the beginning of the day to the end of the day.

Requiring an exact difference can make the problem infeasible. A wider window of $B_{t a}$ and $B_{t d}$ makes the existence of a feasible solution more likely. Another option which would avoid the 
infeasibility issue, could be to introduce a penalty for violation of the balance constraints. The objective would in that case be:

$$
\text { IP3: } \min \sum_{p \in P} c_{p} y_{p}+\sum_{t \in T} c_{e t} e_{t}+c_{m t} m_{t}
$$

where $c_{e t}$ is the unit penalty for having less empty containers at terminal $t$, and $c_{m t}$ is the unit penalty for having more empty containers at terminal $t$ than the required balance $B_{t d}$. The variables $e_{t}$ and $m_{t}$ denote the number of empty containers less than $B_{t a}$ and the number of empty containers more than $B_{t d}$ at terminal $t$, respectively. Softer balance requirement would then be accomplished by replacing constraints (11) and (12) with constraints:

$$
\begin{array}{ll}
\sum_{p \in P}\left(\psi_{t p_{+}} y_{p}-\psi_{t p_{-}} y_{p}\right)-e_{t} \leq B_{t a} & t \in T \\
\sum_{p \in P}\left(\psi_{t p_{+}} y_{p}-\psi_{t p_{-}} y_{p}\right)+m_{t} \geq B_{t d} & t \in T
\end{array}
$$

Constraints (15) ensure that a penalty is paid if the difference in the amount of empty containers between the start of the day to the end of the day is greater than $B_{t a}$. This penalty depends on the amount the $B_{t a}$ is violated by. Constraints (16) is the same ensuring a penalty if the difference is less than $B_{t d}$. In this case the challenge is to determine the value of the penalties $c_{e t}$ and $c_{m t}$.

\section{Test Instances and Computational Results}

The algorithm has been tested on real-life data covering one month of orders in a European country.

\subsection{Test Instances}

In order to test the algorithm, we obtained one month of daily pre- and end-haulage orders from a major liner shipping company, covering a single country in Europe. On weekends the amount of dry container orders is too small to use for test cases hence these days have been removed from the tests. The specific properties of the dry container orders are described in Table 2. The data contains the following characteristics:

- 12 to 308 orders per day

- 10 terminals at 10 different locations for empty container stowage

Each order consists of one container which is either an export order or an import order.

In the given data the origin and destination of the full container, and the storage location of the empty container is provided in geographic coordinates. Moreover, for each order a service time window is defined. These times are usually requested by the customers when they place an import or export order. Customer time windows are often as small as 15 minutes in the given data.

The travel time is calculated based on an estimated average travel speed of $70 \mathrm{~km} / \mathrm{h}$ for the trucks. The wait cost is set to 50 units per hour. 
Note that in this experiment we do not consider handling costs. The path cost is related only to the time used on travel and waiting. Moreover, the gate and wait costs are estimates as we were not provided with exact prices from the company and the cost in real-life would vary depending on container, truck type, time of day and terminal.

We only consider dry containers since refrigerated containers can not be triangulated due to the need of cleaning before being refilled. The four combinations of length and type of the containers considered in the problem are:

- $20 \mathrm{ft}$ normal, $40 \mathrm{ft}$ normal, $20 \mathrm{ft}$ high cube, $40 \mathrm{ft}$ high cube

An import order can be triangulated with an export order if the combination of length and type/height is the same for the containers in both orders, or if the length is the same for containers in both orders and the container of the import order is of type high cube. However, if the import container type is normal height and export container type is high cube then there would not be enough space in the container for the export order, therefore this is not allowed.

\begin{tabular}{|l|r|r|r|}
\hline Date & Orders & Import & Export \\
\hline 03 & 67 & 21 & 46 \\
04 & 206 & 74 & 132 \\
05 & 225 & 70 & 155 \\
06 & 256 & 70 & 186 \\
07 & 247 & 72 & 175 \\
10 & 268 & 83 & 185 \\
11 & 284 & 94 & 190 \\
12 & 12 & 11 & 1 \\
13 & 236 & 48 & 188 \\
14 & 308 & 101 & 207 \\
17 & 244 & 65 & 179 \\
18 & 246 & 71 & 175 \\
19 & 221 & 52 & 169 \\
20 & 242 & 55 & 187 \\
21 & 249 & 50 & 199 \\
24 & 207 & 44 & 163 \\
25 & 208 & 37 & 171 \\
26 & 216 & 43 & 173 \\
27 & 232 & 50 & 182 \\
28 & 263 & 57 & 206 \\
31 & 211 & 46 & 165 \\
01 & 17 & 17 & 0 \\
02 & 158 & 60 & 98 \\
\hline
\end{tabular}

Table 2: Data only containing orders with containers of type HDRY (high cube) and DRY.

From Table 2 it can be seen that on most days there is a majority of export orders. It is frequently the case that there is an imbalance between import and export orders due to the imbalances in world trade. Such imbalances clearly affect the number of triangulations as this number cannot be larger than the minimum number of the two order types. Since orders are placed up to the day of operation, the company requested that the optimal combination of triangulated and single routes must be found in less than a minute to make it a dynamic tool. 


\subsection{Computational Results}

Solution times are not reported since all instances were solved in less than a minute by use of column enumeration and plain CPLEX for the integer programming. The some of the different versions and extensions of the model described in Section 3 are tested.

\begin{tabular}{|c|c|c|c|c|c|c|c|c|c|c|}
\hline \multirow[b]{3}{*}{ Date } & \multicolumn{4}{|c|}{ Predefined empty location } & \multicolumn{4}{|c|}{ Free empty location } & \multirow{2}{*}{\multicolumn{2}{|c|}{$\begin{array}{l}\text { Improvement } \\
\text { using Free } \\
\text { empty location }\end{array}$}} \\
\hline & $\begin{array}{l}\text { Cost } \\
\text { with }\end{array}$ & Imprc & ment & $\begin{array}{c}\text { number } \\
\text { of }\end{array}$ & $\begin{array}{l}\text { Cost } \\
\text { with }\end{array}$ & impr & ment & $\begin{array}{c}\text { number } \\
\text { of }\end{array}$ & & \\
\hline & triang & cost & $\%$ & triang & triang & cost & $\%$ & triang & cost & $\%$ \\
\hline 03 & 15686 & 108 & $1 \%$ & 6 & 12228 & 84 & $1 \%$ & 4 & 3458 & $22 \%$ \\
\hline 04 & 54959 & 3919 & $7 \%$ & 24 & 46446 & 1474 & $3 \%$ & 17 & 8513 & $15 \%$ \\
\hline 05 & 45798 & 4921 & $10 \%$ & 31 & 39094 & 2380 & $6 \%$ & 28 & 6704 & $15 \%$ \\
\hline 06 & 59366 & 9411 & $14 \%$ & 36 & 52149 & 6179 & $11 \%$ & 33 & 7217 & $12 \%$ \\
\hline 07 & 52401 & 3925 & $7 \%$ & 38 & 46387 & 1762 & $4 \%$ & 33 & 6014 & $11 \%$ \\
\hline 10 & 64243 & 9389 & $13 \%$ & 33 & 56635 & 6707 & $11 \%$ & 33 & 7608 & $12 \%$ \\
\hline 11 & 60086 & 9988 & $14 \%$ & 42 & 53626 & 6774 & $11 \%$ & 38 & 6460 & $11 \%$ \\
\hline 12 & 3102 & 0 & $0 \%$ & 0 & 2895 & 0 & $0 \%$ & 0 & 207 & $7 \%$ \\
\hline 13 & 54504 & 6524 & $11 \%$ & 32 & 48105 & 3385 & $7 \%$ & 30 & 6399 & $12 \%$ \\
\hline 14 & 65241 & 9961 & $13 \%$ & 50 & 58963 & 6981 & $11 \%$ & 47 & 6278 & $10 \%$ \\
\hline 17 & 60059 & 5257 & $8 \%$ & 26 & 52388 & 3050 & $6 \%$ & 23 & 7671 & $13 \%$ \\
\hline 18 & 56287 & 5645 & $9 \%$ & 33 & 49615 & 3746 & $7 \%$ & 31 & 6672 & $12 \%$ \\
\hline 19 & 55819 & 3926 & $7 \%$ & 24 & 47672 & 1801 & $4 \%$ & 20 & 8147 & $15 \%$ \\
\hline 20 & 62011 & 3849 & $6 \%$ & 25 & 54052 & 2564 & $5 \%$ & 26 & 7959 & $13 \%$ \\
\hline 21 & 67580 & 8568 & $11 \%$ & 33 & 60244 & 6407 & $10 \%$ & 30 & 7336 & $11 \%$ \\
\hline 24 & 46546 & 2835 & $6 \%$ & 17 & 38624 & 1289 & $3 \%$ & 14 & 7922 & $17 \%$ \\
\hline 25 & 55442 & 2163 & $4 \%$ & 13 & 46299 & 1173 & $2 \%$ & 10 & 9143 & $16 \%$ \\
\hline 26 & 57048 & 4847 & $8 \%$ & 19 & 50669 & 3240 & $6 \%$ & 20 & 6379 & $11 \%$ \\
\hline 27 & 58811 & 5139 & $8 \%$ & 30 & 49962 & 3533 & $7 \%$ & 24 & 8849 & $15 \%$ \\
\hline 28 & 74528 & 6040 & $7 \%$ & 38 & 65866 & 2807 & $4 \%$ & 35 & 8662 & $12 \%$ \\
\hline 31 & 49634 & 1583 & $3 \%$ & 18 & 40648 & 804 & $2 \%$ & 13 & 8986 & $18 \%$ \\
\hline 01 & 3485 & 0 & $0 \%$ & 0 & 3485 & 0 & $0 \%$ & 0 & 0 & $0 \%$ \\
\hline 02 & 41383 & 2088 & $5 \%$ & 6 & 35058 & 1553 & $4 \%$ & 6 & 6325 & $15 \%$ \\
\hline
\end{tabular}

Table 3: Data only containing orders with containers of type HDRY and DRY. For the triangulations a wait cost has been introduced so that the cost is zero for the first half an hour and hereafter 50 units per hour. The speed is assumed to be $70 \mathrm{~km} / \mathrm{h}$, and a cost of 70 units per hour is used as travel cost. Here the model IP1 is applied.

Table 3 reports the results of routing without triangulation compared to routing with the possibility of triangulation. Two versions of the problem are considered: one is the case where the location for picking up and delivering an empty container is predefined for simple routes, the other were the location for picking up and delivering the empty container can be chosen freely among a set of locations. All of the solutions in Table 3 were found by enumerating all feasible paths and using CPLEX for selecting the combination of routes. The optimal solution was in all test cases found in less than a second. Column 1 in Table 3 denotes the date for which data was received. Columns 2, 3, 4 and 5 show the case where the empty container location of each order is given in the data. Column 2 shows the total cost for the day allowing triangulation and column 5 shows 
the number of triangulations used to achieve this solution. Column 3 shows the amount by which the solution is reduced by allowing triangulations and column 4 shows the reduction in percent. Columns 6, 7, 8 and 9 consider the case where the empty container location is free to be selected among the set of terminals. In this set there are 10 terminals form which an empty container can be picked up or delivered. Column 6 shows the cost for the day of operations when optimizing triangulations. Column 7 shows the reduction in cost achieved by using triangulations on this version of the problem and column 8 shows the reduction in percent. Column 9 shows the number of triangulations used in the solution presented in column 6. Column 10 shows the difference in solution value between using free locations and predefined locations while column 11 contains the percent reduction between the solution with predefined location (column 2) and the solution with free locations (column 6) for the case where triangulations are permitted. It is shown in Table 3 that the minimal solution using triangulations may save up to $14 \%$ of the daily operation cost. Note that the savings are slightly smaller in the case of free empty container depot as there is less saving to be achieved on the empty container journey in this case.

From Table 3 it is clear that having free empty container location can improve the solution with up to $22 \%$ and 50 triangulations. It is impossible for the company to manually find the optimal combination of data sets this size. However, from the company perspective this may not be as attractive as it seems, since the solutions may cause containers to be unevenly distributed and result in the need of repositioning of empty containers for which cost is not considered here. A way to avoid this is to introduce constraints on the balance in the removal and depositing of containers at each terminal. This can be done by using the constraints presented in Section 3.4. Note that the number of paths $P$ in this case is only a couple of thousands as the paths at most contains two orders and the time window constraints can eliminate quite a few.

\subsubsection{Test results for container yard balancing}

Table 4 reports the results for instances where the inventory balance must stay within an upper and lower limit at each terminal. The test is done for hard yard balancing constraints. Let $\Delta$ be the maximum number of containers that every terminal is allowed to increase or decrease their inventory by, at the end of the day. This means that $\Delta=B_{t a}=-B_{t d}$. The empty container location can be selected freely among the 10 available terminals/depot locations.

The test instances have been solved with parameters $\Delta=15, \Delta=20$ and $\Delta=30$. It is clear that the overall cost should decrease when $\Delta$ increases. These can also be compared to columns 6 and 9 in the Table 3 which represents the unbounded case.

In Table 4 the first column lists the test instances. Column 2 and 3 show respectively the cost and the number of triangulations of a minimum cost solution for $\Delta=15$ for all terminals. Column 4 and 5 show the same for the case where $\Delta$ increased to 20 . In columns 6 and $7 \Delta$ is increased to 30 and in column 8 and $9, \Delta$ is unbounded for all terminals. Note that if the total balance does not satisfy the difference between the import and export and there are not enough feasible triangulations there may not be a feasible solution. From the tests it is clear that a terminal restriction does increase the cost significantly as $\Delta$ decreases. However, a company should use this to compare with the cost and need of repositioning. From the unbounded case to $\Delta=15$ instance 25 increases with more than $\$ 50000$ which is also more than $50 \%$ of the cost. This shows that these restrictions are quite costly and should only be used if necessary. Again for Table 


\begin{tabular}{|c|c|c|c|c|c|c|}
\hline & \multicolumn{2}{|c|}{15} & \multicolumn{2}{|c|}{20} & \multicolumn{2}{|c|}{30} \\
\hline Date & $\begin{array}{c}\text { Cost } \\
\text { with } \\
\text { triangulate }\end{array}$ & $\begin{array}{c}\text { Number } \\
\text { of } \\
\text { triang }\end{array}$ & $\begin{array}{c}\text { Cost } \\
\text { with } \\
\text { triangulate }\end{array}$ & $\begin{array}{c}\text { Number } \\
\text { of } \\
\text { triang }\end{array}$ & $\begin{array}{c}\text { Cost } \\
\text { with } \\
\text { triangulate }\end{array}$ & $\begin{array}{c}\text { Number } \\
\text { of } \\
\text { triang }\end{array}$ \\
\hline 03 & 13329 & 5 & 12806 & 5 & 12228 & 3 \\
\hline 04 & 53174 & 18 & 49300 & 17 & 48137 & 17 \\
\hline 05 & 55351 & 31 & 47960 & 31 & 41037 & 31 \\
\hline 06 & 82367 & 33 & 70261 & 34 & 56380 & 33 \\
\hline 07 & 70493 & 30 & 60405 & 28 & 48167 & 30 \\
\hline 10 & 78414 & 31 & 68377 & 32 & 59527 & 32 \\
\hline 11 & 68307 & 41 & 59494 & 39 & 54904 & 43 \\
\hline 12 & 2895 & 0 & 2895 & 0 & 2895 & 0 \\
\hline 13 & 84868 & 28 & 69239 & 30 & 51867 & 29 \\
\hline 14 & 84984 & 45 & 73470 & 46 & 62274 & 48 \\
\hline 17 & 75791 & 23 & 64411 & 23 & 53521 & 25 \\
\hline 18 & 73461 & 29 & 63103 & 29 & 53160 & 31 \\
\hline 19 & 78707 & 20 & 66289 & 21 & 52586 & 22 \\
\hline 20 & 91384 & 23 & 77537 & 24 & 58857 & 33 \\
\hline 21 & 102603 & 25 & 86408 & 26 & 66638 & 28 \\
\hline 24 & 76108 & 12 & 63223 & 13 & 47627 & 13 \\
\hline 25 & 102800 & 10 & 86854 & 14 & 66651 & 12 \\
\hline 26 & 89274 & 20 & 74125 & 21 & 57719 & 20 \\
\hline 27 & 82744 & 24 & 68788 & 24 & 52680 & 24 \\
\hline 28 & 104911 & 31 & 89759 & 31 & 70740 & 35 \\
\hline 31 & 79858 & 13 & 67330 & 13 & 51575 & 16 \\
\hline 01 & 3495 & 0 & 3485 & 0 & 3485 & 0 \\
\hline 02 & 38817 & 6 & 35939 & 6 & 35359 & 6 \\
\hline
\end{tabular}

Table 4: The results with limitations on the difference at the terminal. The test is done with 10 empty container terminals. The cases are where each terminal allows at the end of the day to have between 15 containers less and 15 containers more than in the morning. The same is repeated for 20 and 30 containers.

4 it should be noted that the optimal solutions on all test sets were found in less than a second.

\subsubsection{Test results for minimizing number of vehicles}

In Table 5 we consider the variant of the problem where the number of vehicles is minimized. As mentioned earlier in the case where the transports are carried out by subcontractors the number of vehicles used does not add cost to the company receiving the orders as the subcontractors will charge per order based on distance and time while the subcontractors themselves optimize their vehicle usage in combination with orders from other companies as well. However, there may exist some situations where the companies have their own fleets of trucks. In this case reducing the number of vehicles may decrease cost of operation. Moreover this is investigated in previous literature such as Imai et al. [4] and Caris and Janssens [3] and therefore a variant of this case is considered here. We wished to see if the algorithm would still find an optimal solution in less than a minute for all of our test instances. 


\begin{tabular}{|c|c|c|c|c|c|c|c|}
\hline \multirow[b]{2}{*}{ Date } & \multicolumn{3}{|c|}{ Max triangulation } & \multicolumn{2}{|c|}{ Fixed empty } & \multicolumn{2}{|c|}{ Semi free empty } \\
\hline & Cost & $\begin{array}{r}\text { Nun } \\
0 \\
\text { vehicles }\end{array}$ & & Cost & $\begin{array}{l}\text { Number } \\
\text { of } \\
\text { vehicles }\end{array}$ & Cost & $\begin{array}{l}\text { Number } \\
\text { of } \\
\text { vehicles }\end{array}$ \\
\hline 03 & 15747 & 61 & 6 & 15746 & 53 & 15329 & 51 \\
\hline 04 & 55402 & 180 & 26 & 55170 & 144 & 51219 & 135 \\
\hline 05 & 46090 & 188 & 37 & 46204 & 130 & 44777 & 127 \\
\hline 06 & 60553 & 211 & 46 & 59769 & 148 & 58709 & 144 \\
\hline 07 & 53608 & 202 & 46 & 52444 & 145 & 51256 & 143 \\
\hline 10 & 64964 & 229 & 40 & 64292 & 184 & 63255 & 181 \\
\hline 11 & 62052 & 224 & 60 & 60120 & 162 & 57620 & 155 \\
\hline 12 & 3102 & 12 & 0 & 3311 & 10 & 3311 & 10 \\
\hline 13 & 55492 & 195 & 41 & 55330 & 142 & 53700 & 139 \\
\hline 14 & 65632 & 249 & 59 & 65509 & 185 & 64257 & 182 \\
\hline 17 & 60603 & 211 & 34 & 60349 & 145 & 56934 & 142 \\
\hline 18 & 56695 & 209 & 38 & 56883 & 151 & 56710 & 150 \\
\hline 19 & 56504 & 189 & 32 & 56083 & 133 & 54822 & 133 \\
\hline 20 & 62892 & 208 & 34 & 62341 & 156 & 61212 & 152 \\
\hline 21 & 68576 & 212 & 40 & 67867 & 169 & 66785 & 166 \\
\hline 24 & 46725 & 187 & 20 & 46870 & 129 & 45458 & 126 \\
\hline 25 & 58962 & 186 & 24 & 55868 & 138 & 54193 & 136 \\
\hline 26 & 58958 & 183 & 33 & 57224 & 149 & 55141 & 141 \\
\hline 27 & 58940 & 200 & 32 & 58968 & 156 & 56957 & 152 \\
\hline 28 & 75278 & 217 & 49 & 74659 & 181 & 71794 & 175 \\
\hline 31 & 50827 & 186 & 25 & 49857 & 142 & 49229 & 140 \\
\hline 01 & 3485 & 17 & 0 & 3515 & 11 & 3515 & 11 \\
\hline 02 & 41383 & 152 & 6 & 41383 & 138 & 41317 & 138 \\
\hline
\end{tabular}

Table 5: The results when minimizing the number of vehicles. The distance and travel time is included in the objective to ensure the selection of the best possible solution with respect to distance and travel time with the minimal set of vehicles. 3 different ways of arranging a route for a vehicle is presented. Max triangulation: A return trip to a terminal corresponds to a vehicle, this case will maximize the number of triangulations. Fixed empty: The return trips are combined so that a vehicle can do more than one return trip if time allows. Semi-free empty: The empty container may be deposited at a terminal where a full container is picked up and the empty container for the next order may be picked up where the full container is deposited. All tests were solved in less than a minute.

We have constructed three different versions of the problem of minimizing the number of vehicles. One is the simple case where the same routes as earlier are generated not adding more variables to the problem. In this case each vehicle represents a single trip which ends when entering a terminal and minimizing the number of vehicles corresponds to maximizing the number of triangulations. Therefore this result will provide us with the maximum number of triangulations possible in a solution for each instance. All of these tests were solved in less than a second.

The second problem is to combine the single trips while satisfying the time windows. Note that when combining the trips, a wait cost is introduced if there is some time to wait between trips. If one trip ends at one terminal and the next starts at another terminal a travel edge and cost is 
added to the route sending the vehicle from one terminal to the next. The trips combined are the trips using predefined empty container locations and triangulations.

The third problem is to modify slightly on the second problem by allowing an empty container to be deposited at the terminal where the next trip starts and an empty container to be picked up at the terminal where the last trip ends. This is defined here as having a semi free empty location. In column 1 of Table 5 the test instances are listed. Columns 2, 3 and 4 contain the data for the first version of the problem where the number of triangulations in a solution is maximized. Column 2 shows the travel and wait time cost, column 3 shows the number of vehicles used and column 4 shows the number of triangulations in the solution. Even though the number of vehicles is minimized for all of the three problems presented the cost shown is the minimal total travel cost when using the minimal number of vehicles.

Columns 5 and 6 show the results of the second version of the problem where the single or triangulated routes are combined while satisfying the time window constraints. Column 5 shows the travel and wait time cost of the optimal solution and column 6 shows the number of vehicles needed in the optimal solutions of the second version. Note that in the second version the locations of the empty containers are predefined. Columns 7 and 8 show the results for the optimal solution when the position of empty containers can be adjusted to fit the route combined with. Column 7 shows the travel and wait time cost of the optimal solution and column 8 shows the number of vehicles needed in the optimal solution.

The results in Table 5 show that the version with semi free empty container locations reduces the number of vehicles needed and in most cases also improves the travel and waittime costs. Note that all three problem variants include the possibility of triangulation. All test cases were solved in less than a minute for the second and third versions of the problem.

\subsubsection{Import and export specific time windows}

For the test shown in Table 3, 4 and 5 the time windows were provided by the liner shipping company. However, one might wish to fix the time windows using some strategy which may reduce the overall cost. Since an import costumer must be visited before an export costumer when triangulating, we have tested the instances with a fixed morning time for the imports and a fixed afternoon time for the exports. Results of these tests are shown in Table 6. Nearly all of the 23 test cases show an improvement of up to 5\%. In one test case a slight increase in cost of $1 \%$ occurs and in one test case an decrease in cost of $15 \%$ is achieved by restricting the time window. It can be seen by the last column in Table 6 that the rule may not always result in a solution with more triangulations. In those cases the triangulations the average cost reduction on each triangulations is larger.

The results shown in Table 6 show that fixing the imports to $10 \mathrm{am}$ and the exports to $3 \mathrm{pm}$ in most cases will reduce the cost. However, the possible customer dissatisfaction of operating with fixed time windows for different order directions needs to be considered before implementing such operation rules. 


\begin{tabular}{|c|c|c|c|c|c|c|c|}
\hline \multirow[b]{2}{*}{ Date } & \multicolumn{2}{|c|}{ Given time window } & \multicolumn{2}{|c|}{ Import 10am Export 15pm } & \multicolumn{3}{|c|}{ Improvements } \\
\hline & $\begin{array}{l}\text { Cost } \\
\text { with } \\
\text { triangulate }\end{array}$ & $\begin{array}{c}\text { number } \\
\text { of } \\
\text { triangulate }\end{array}$ & $\begin{array}{l}\text { Cost } \\
\text { with } \\
\text { triangulate }\end{array}$ & $\begin{array}{l}\text { number } \\
\text { of } \\
\text { triangulate }\end{array}$ & cost & percent & $\begin{array}{l}\text { triangulate } \\
\text { diff }\end{array}$ \\
\hline 03 & 15686 & 6 & 15381 & 4 & 305 & $2 \%$ & -2 \\
\hline 04 & 54959 & 24 & 52234 & 34 & 2725 & $5 \%$ & 10 \\
\hline 05 & 45798 & 31 & 45612 & 28 & 186 & $0 \%$ & -3 \\
\hline 06 & 59366 & 36 & 58271 & 41 & 1095 & $2 \%$ & 5 \\
\hline 07 & 52401 & 38 & 50944 & 31 & 1457 & $3 \%$ & -7 \\
\hline 10 & 64243 & 33 & 62653 & 50 & 1590 & $2 \%$ & 17 \\
\hline 11 & 60086 & 42 & 57864 & 54 & 2222 & $4 \%$ & 12 \\
\hline 12 & 3102 & 0 & 3102 & 0 & 0 & $0 \%$ & 0 \\
\hline 13 & 54504 & 32 & 54830 & 21 & -326 & $-1 \%$ & -11 \\
\hline 14 & 65241 & 50 & 64141 & 49 & 1100 & $2 \%$ & -1 \\
\hline 17 & 60059 & 26 & 57156 & 26 & 2903 & $5 \%$ & 0 \\
\hline 18 & 56287 & 33 & 54425 & 38 & 1862 & $3 \%$ & 5 \\
\hline 19 & 55819 & 24 & 53616 & 19 & 2203 & $4 \%$ & -5 \\
\hline 20 & 62011 & 25 & 58747 & 32 & 3264 & $5 \%$ & 7 \\
\hline 21 & 67580 & 33 & 65514 & 33 & 2066 & $3 \%$ & 0 \\
\hline 24 & 46546 & 17 & 45955 & 19 & 591 & $1 \%$ & 2 \\
\hline 25 & 55442 & 13 & 53761 & 15 & 1681 & $3 \%$ & 2 \\
\hline 26 & 57048 & 19 & 54897 & 21 & 2151 & $4 \%$ & 2 \\
\hline 27 & 58811 & 30 & 56026 & 31 & 2785 & $5 \%$ & 1 \\
\hline 28 & 74528 & 38 & 72343 & 38 & 2185 & $3 \%$ & 0 \\
\hline 31 & 49634 & 18 & 48748 & 18 & 886 & $2 \%$ & 0 \\
\hline 01 & 3485 & 0 & 3485 & 0 & 0 & $0 \%$ & 0 \\
\hline 02 & 41383 & 6 & 34988 & 25 & 6395 & $15 \%$ & 19 \\
\hline
\end{tabular}

Table 6: Tests of the effects of changing the time window. The results on the initial time windows provided in data are shown and compared to the results of changing the time windows so that imports occur at $10 \mathrm{am}$ and exports at $3 \mathrm{pm}$. These times are chosen in the hope that fixing imports to morning hours and exports to afternoon hours will provide more triangulations and thereby a reduction in the overall cost.

\section{Conclusion}

In this paper we have considered an intermodal transportation problem arising in the liner shipping industry. We have proposed several different mathematical models for optimizing a one-day schedule covering all import and export orders. The presented model finds the best combination of routes from a complete enumeration of paths. In the experiments we have considered singlelocation paths and triangulation paths. The model has been tested on instances covering 23 days from a liner shipping company, and we have analyzed the solution quality. Due to the tight time windows of the considered data, the imbalance between imports and exports, and the problem of matching the container sizes and type, the number of possible triangulations is quite limited. This makes it possible to find an optimal solution within seconds for the problem of the liner shipping company and other versions not minimizing the number of vehicles. This is even for instances with more than 300 orders. 
However, significant cost reductions are still obtained using triangulation. Different models for selecting the empty container depots have been considered, and constraints for limiting any imbalance has been formulated. The effect of such limits on the routing cost has been investigated. Significant savings using triangulations have been shown.

It has been shown that for the considered real-life problems, path enumeration is a manageable approach for finding optimal solutions. Due to the short calculation time, a program using the enumeration method has been implemented in VBA as an Excel add-in using Opensolver 2.1. The Excel add-in finds the optimal solution in less than a minute for the test cases and is currently being tested at the liner shipping company.

\section{References}

[1] K. Braekers, A. Caris, and G.K. Janssens. A deterministic annealing algorithm for a biobjective full truckload vehicle routing problem in drayage operations. Procedia-Social and Behavioral Sciences, 20:344-353, 2011.

[2] K. Braekers, A. Caris, and G.K. Janssens. Integrated planning of loaded and empty container movements. OR Spectrum, 35(2):457-478, 2013.

[3] A. Caris and G.K. Janssens. A local search heuristic for the pre- and end-haulage of intermodal container terminals. Computers and Operations Research, 36(10):2763-2772, 2009.

[4] A. Imai, E. Nishimura, and J. Current. A lagrangian relaxation-based heuristic for the vehicle routing with full container load. European Journal of Operational Research, 176:87-105, 2007.

[5] H. Jula, M. Dessouky, P. Ioannou, and A. Chassiakos. Container movement by trucks in metropolitan networks: Modeling and optimization. Transportation Research Part E: Logistics and Transportation Review, 41(3):235-259, 2005.

[6] A. Mingozzi, S. Giorgi, and R. Baldacci. Exact method for the vehicle routing problem with backhauls. Transportation Science, 33:315-329, 1999.

[7] J. Nossack and E. Pesch. A truck scheduling problem arising in intermodal container transportation. European Journal of Operational Research, 230(3):666-680, 2013.

[8] I.H. Osman and N.A. Wassan. A reactive tabu search meta-heuristic for the vehicle routing problem with back-hauls. Journal of Scheduling, 5:263-285, 2002.

[9] L.B. Reinhardt, S. Spoorendonk, and D. Pisinger. Solving vehicle routing with full container load and time windows. In Computational Logistics, volume LNCS 7555, pages 120-128. Springer Berlin Heidelberg, 2012.

[10] J. Schönberger, T. Buer, and H. Kopfer. A model for the coordination of 20-foot and 40-foot container movements in the hinterland of a container terminal. In Computational Logistics, LNCS, vol 8197, pages 113-127. Springer Berlin Heidelberg, 2013. 
[11] P. Toth and D. Vigo. An exact algorithm for the vehicle routing problem with backhauls. Transportation Science, 31:372-385, 1997.

[12] M. Vidovic, G. Radivojevic, and B. Rakovic. Vehicle routing in containers pickup up and delivery processes. Procedia - Social and Behavioral Sciences, 20:335-343, 2011.

[13] W.F. Wang and W.Y. Yun. Scheduling for inland container truck and train transportation. International Journal of Production Economics, 143(2):349-356, 2013.

[14] X. Wang and A.C. Regan. Local truckload pickup and delivery with hard time window constraints. Transportation Research Part B: Methodological, 36(2):97-112, 2002.

[15] G. Zhang, K. Smilowitz, and A. Erera. Dynamic planning for urban drayage operations. Transportation Research Part E: Logistics and Transportation Review, 47(5):764-777, 2011.

[16] R. Zhang, W.Y. Yun, and H. Kopfer. Heuristic-based truck scheduling for inland container transportation. OR Spectrum, 32(3):787-808, 2010. 